\title{
Cryo-EM Structure of GroEL in Complex with the Prion Protein
}

Evgeny Pichkur ${ }^{1}$, Sofia Kudryavtseva ${ }^{2}$, Olga Sokolova ${ }^{2}$ and Tatiana Stanishneva-Konovalova ${ }^{2}$

${ }^{1}$ Kurchatov Institute, Moscow, Moskva, Russia, ${ }^{2}$ Lomonosov Moscow State University, Faculty of Biology, Moscow, Moskva, Russia

A number of severe neurodegenerative disorders, including Parkinson's, Alzheimer's, and prion diseases, are associated with the accumulation of protein aggregates in healthy tissues [1]. Aggregation of proteins occurs due to the disruption of their secondary structure, which can be genetically determined [2], and caused by the influence of metal ions [3], acidification [4] or other factors. This leads to the formation of oligomers that can permeate the cell and are highly toxic [5]. In cells, protein aggregates meet with local defense systems. One of these cellular defense systems is the chaperone system, which regulates the correct folding of proteins, prevents their aggregation, and is even capable of destroying already formed aggregates [6]. Therefore, one of the experimental directions in the treatment and prevention of neurodegenerative diseases is the use of chaperone systems [7]. However, the outcome of the interaction of chaperones with an amyloidogenic protein and its aggregates may be different. On the one hand, the chaperone can help the target protein to acquire the native conformation. On the other hand, it can dissect large oligomers to smaller ones, which will lead to the emergence of new spots of aggregation and will accelerate the development of the disease [8]. In this project, we used cryo-EM to study the formation of a complex between GroEL, as a well-studied representative of chaperone family, and an amyloidogenic prion protein $(\mathrm{PrP})$.

To confirm the formation of the GroEL-PrP complex, the dynamic light scattering (DLS) method was implemented. Using the Zetasizer Nano-ZS instrument, we measured the particle sizes in a PrP solution $(3 \mu \mathrm{M})$, in a GroEL solution $(3 \mu \mathrm{M})$, and after mixing the proteins in a 1:1 molar ratio. For the GroELPrP mixture, measurements were carried out immediately after mixing and then after 10, 20, 30, and 40 minutes of co-incubation with constant stirring. Figure 1 demonstrates particle size distribution at the beginning of the experiment and after 40 minutes of incubation. Each distribution shown in the graphs represents the average of 3 measurements taken over 50 seconds. The DLS results indicate that, upon mixing of the two proteins, the GroEL-PrP complex is formed, since a new peak appears with a hydrodynamic diameter of $16 \mathrm{~nm}$, different from the peaks of pure prion protein $(4 \mathrm{~nm})$ and pure GroEL $(12 \mathrm{~nm})$. The complex was present in solution at the beginning of measurements and after 40 minutes. In addition, particles with a diameter of $500-700 \mathrm{~nm}$ were detected after 40 minutes of incubation, which may be caused by PrP aggregation. Confirming the formation of the GroEL-PrP complex, we proceeded to study its structure by cryo-electron microscopy.

In order to precipitate the aggregates, a sample containing $8 \mu \mathrm{M}$ GroEL and $16 \mu \mathrm{M} \operatorname{PrP}$ (in $50 \mathrm{mM}$ Tris$\mathrm{HCl}$ buffer, $\mathrm{pH}$ 7.5, $1 \mathrm{mM}$ EDTA) was centrifuged for $5 \mathrm{~min}$ at $12000 \mathrm{rpm}$, and then the supernatant was concentrated 2 times on a Millipore Microcon with a filter of $100 \mathrm{kDa}$ (from 50 to $25 \mu \mathrm{l}$ ). Quantifoil grids were glow discharged in a PELCO easiGlow system, then $3 \mu \mathrm{l}$ of a GroEL-PrP sample was applied to a grid and vitrified in a chamber of the Vitrobot Mark IV apparatus. Frozen grids were transferred to a Titan Krios 60-300 cryo-electron microscope equipped with a Falcon II electron detector, where images were taken using the EPU (FEI) software (1631 image stacks in total). Image processing and 3D reconstruction were carried out using the Warp [9] and CryoSPARC [10] software packages. After initial particle selection and two-dimensional classification, 137319 particles were selected for further processing. The selected particles were subjected to a three-dimensional classification procedure, which allowed the separation of free GroEL particles from GroEL bound to $\operatorname{PrP}$ (the number of latter was 106260). As a 
result, we obtained the structure of the GroEL-PrP complex (Figure 2) with a resolution of $3.7 \AA$ according to the $\mathrm{FSC}=0.143$ criterion.

Compared to the apo-GroEL structure, the structure of the complex has an additional density at the level of the apical domains. This density is located near the helix I (amino acid residues 256-271) of two neighboring GroEL subunits, as well as near the helix H (residues 230-244) of one of them. The participation of $\mathrm{H}$ and I helices of the apical domains in substrate binding is consistent with available literature data [11]. Although the secondary structure of the additional density is unclear, we suggest that it corresponds to the C-terminal domain of $\mathrm{PrP}$, which in the free state is represented by three alpha helices and two beta strands. The N-terminal domain of PrP is not structured and has a large conformational mobility; therefore, it is expected to be absent from the reconstruction of the complex.

Previously, PrP was demonstrated to inhibit the GroEL-assisted reactivation of the glycolytic enzyme glyceraldehyde-3-phosphate dehydrogenase [8]. Here, we obtained the structure of their complex, showing that PrP binds two neighboring apical domains of GroEL. Our results imply that the ability of PrP or other amyloidogenic proteins to interfere with chaperone functioning may be involved in the development of neurodegenerative diseases [12].

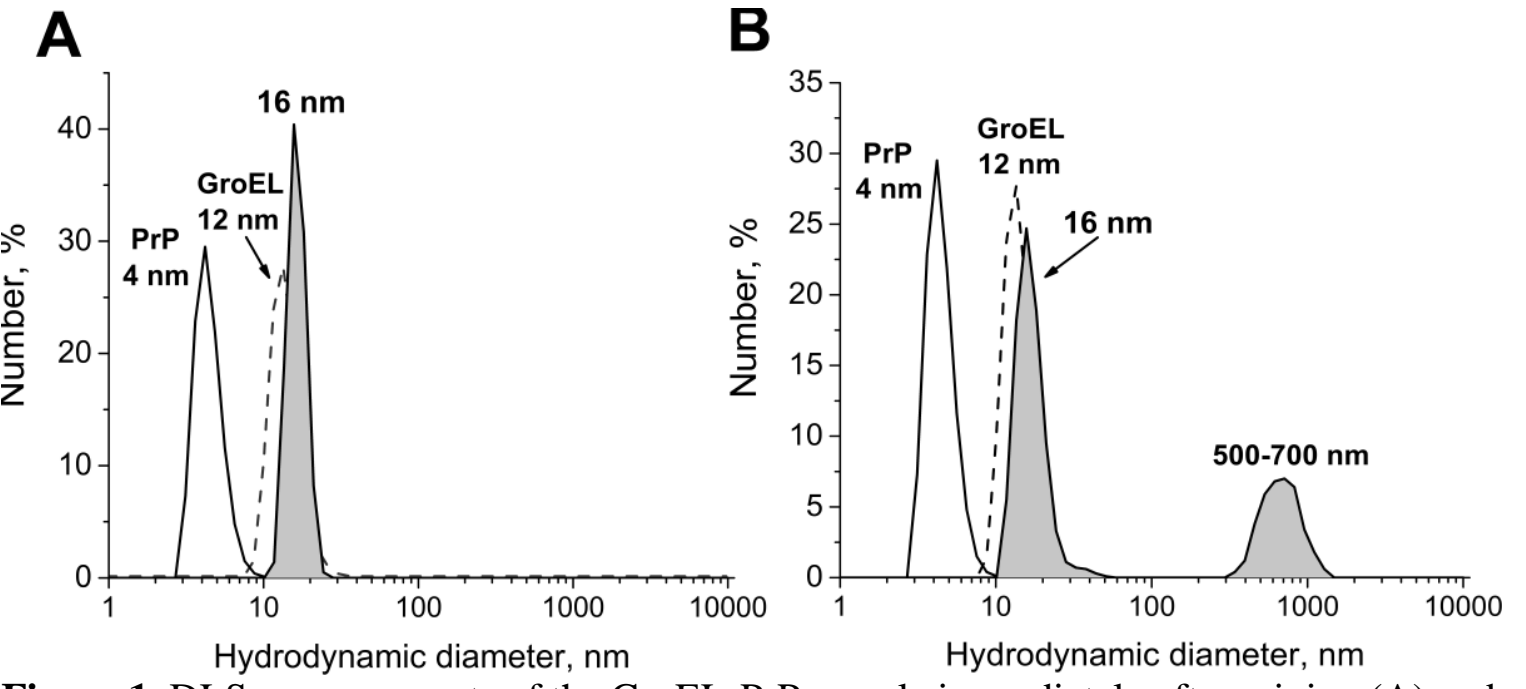

Figure 1. DLS measurements of the GroEL-PrP sample immediately after mixing (A) and after 40 min of incubation (B). The particle size distribution in solutions of pure PrP (solid line), pure GroEL (dotted line), and GroEL-PrP mixture (colored in gray) is shown. 


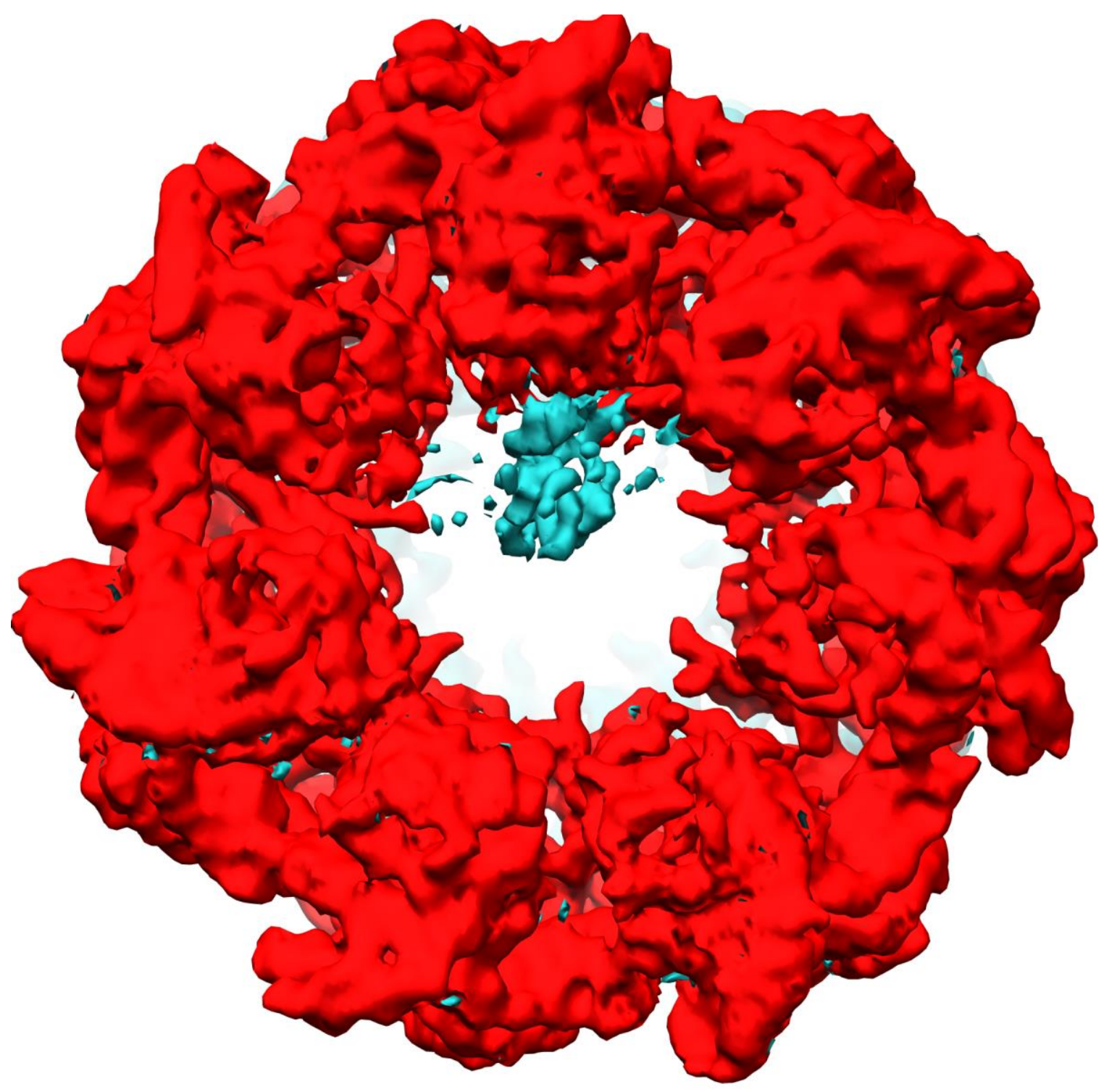

Figure 2. The 3D structure of the GroEL-PrP complex as seen from the apical domains. The part of the structure corresponding to GroEL is colored in red and the additional density corresponding to PrP is colored in turquoise. Bar size $-10 \mathrm{~nm}$.

\section{References}

[1] Iadanza MG, et al (2018) Nat. Rev. Mol. Cell Biol. 19, 755-773

[2] Byers B, et al (2011) PLoS One. 6, e26159

[3] Viles JH (2012) Coord. Chem. Rev. 256, 2271-2284.

[4] Goodchild SC, et al (2014) PLoS One. 9, e104492

[5] Sengupta U, et al (2016) EBioMedicine 6, 42-49.

[6] Bose D \& Chakrabarti A (2017) IUBMB Life 69, 647-659.

[7] Friesen EL, et al (2017) Parkinsons. Dis. 2017. 5015307

[8] Kudryavtseva SS, et al (2016) Biochem. 81, 1213-1220.

[9] Tegunov D \& Cramer P (2019) Nat. Methods 16, 1146-1152.

[10] Punjanji A, et al (2017) Nat. Methods 14, 290-296. 
[11] Elad N, et al (2007) Mol. Cell 26, 415-426.

[12] The authors acknowledge funding from the Russian Science Foundation (grant \#19-74-20055). 